Ann. Zootech., I96I, 10 (3), 205-2I3.

\title{
EFFICACITÉ COMPARÉE POUR LA GROISSANCE DU POUSSIN DE LA MÉTHIONINE ET DU SEL DE CALCIUM DE L'ACIDE HYDROXYMÉTHYLTHIOBUTYRIQUE (M. H. A.)
}

\author{
C. CALET et M. MELO'T. \\ Station de Recherches azicoles. \\ Centre national de Recherches zootechniques, Jouy-en-Josas (Seine-et-Oise).
}

\section{SOMMAIRE}

\begin{abstract}
Nous avons comparé les aptitudes de la DI.-méthionine et du M. H. A. à valoriser les protides du tourteau de soja cru en employant un plan de rationnement nouveau. Cette méthode consiste à séparer les constituants du régime et à distribuer simultanément dans deux mangeoires différentes d'une part une quantité limitée de matières azotées à étudier et cl'autre part des substances énergétiques non azotées. Ces dernières sont fournies au poussin à volonté de manière à lui laisser libre la consommation des calories nécessaires à la couverture de son besoin énergétique. Dans ces conditions, le M. H. A. possède la même efficacité que la méthionine lorsque ces deux substances sont comparées sur la base du produit actif. On observe la même croissance, la même consommation spontanée d'énergie et la même efficacité des régimes. Par contre, comparé sur la base du produit brut, le M. H. A. s'est révélé toujours inférieur à la méthionine quel que soit le critère de mesure utilisé.
\end{abstract}

On connait plusieurs substances qui interviennent dans le métabolisme de la méthionine. Les unes permettent sa synthèse en fournissant des groupements méthyles libres ; les autres sont des intermédiaires dans les chaînes de réaction qui aboutissent à sa formation. Il existe toutefois des différences selon les espèces animales. Ainsi la diméthylpropiothétine présente chez le rat les mêmes propriétés que la méthionine tant pour permettre l'accumulation de fer dans le foie (CoHEN et al., r958) que pour servir de donateur de méthyle (Maw et du VigneAUD, I948). En revanche, chez le poulet, SAnson et MatTerson, r957, montrent que non seulement la diméthylpropiothétine ne peut remplacer la méthionine, mais au contraire s'oppose à son utilisation lorsque les deux corps sont introduits dans l'aliment.

Au cours de la dégradation métabolique de la méthionine, 1'acide cétométhiolbutyrique se forme normalement par désamination oxydative. Or on connait une 
substance très voisine, l'acide DL 2-hydroxy-4-méthylthiobutyrique, qui diffère du précédent par le remplacement d'une fonction cétonique par une fonction alcool. Depuis quelques années, on a recherché dans quelle mesure cette substance, qui se trouve soit sous forme de sel de calcium soit sous forme d'amide et qui est connue sous le nom de méthyl-hydroxy-analogue de la méthionine (M. H. A.), possède une efficacité comparable à celle-ci. On a signalé plusieurs fois l'intérêt de ces substances qui, en l'absence de méthionine, valorisent aussi bien le régime du poussin (Gordon, MAdDy, KNIGHT, I954; Douglas et al. I957; Gordon et al. I957) que celui de la poule pondeuse (BARTON et STEPHENSON, I960). L'efficacité du M. H. A. a été comparée à celle de la méthionine dans différentes conditions. BIRD, I952, démontre l'égalité de ces deux substances pour la croissance du poussin lorsqu'elles sont apportées à doses égales de produit actif. De la même manière, REID, GABAy et Couch, I954, opérant en culture de tissu, accordent au M. H. A. et à la méthionine la même faculté à relever la teneur du foie en glutathion.

Malgré ces conclusions concordantes, de récents travaux ont remis le problème en question.

La confrontation des résultats de MAchlin et GoRdon, I958 et I959, d'une part, et ceux de Suli,IVAN et BIRD, I957, d'autre part révèle une nette opposition. Les premiers auteurs proclament l'équivalence de la méthionine et du M. H. A. lorsqu'on les compare à égalité de produit brut. Or la DI-méthionine dose $98 \%$ de produit actif alors que le M. H. A. n'en renferme que 80 p. Ioo. Cela voudrait donc dire qu'à égalité de produit actif le M. H. A. possède une efficacité supérieure à celle de la méthionine. Pour Sullivan et BrRd, I957, au contraire, le M. H. A. manifeste une infériorité constante vis-à-vis de la méthionine quel que soit le taux protéique du régime.

Nous avons remarqué (CALET, JOUANDET, BARATOU, I960), que la distribution des protides dans un aliment complet ne représente pas la meilleure méthode pour révéler leur efficacité. Ceci est encore plus marqué lorsque les protéines possèdent une haute valeur biologique. Il convient mieux de distribuer simultanément dans deux mangeoires séparées les constituatıts azotés du régime d'une part et les substances énergétiques non azotées d'autre part. En utilisant cette nouvelle méthode, nous avons cherché à lever les contradictions qui demeurent quant à l'égalité des efficacités de la méthionine et de son analogue.

\section{MATÉRIEI, ET MÉTHODES}

I.expérience est conduite en cages individuelles et pour cela une période préexpérimentale est nécessaire au cours de laquelle les poussins expérimentaux sont choisis et habitués progressivement à vivre seuls. 200 poussins mâles d'un jour issus du croisement Rhode Wyandotte sont répartis en 50 cages. Pendant 2 I jours, on élimine $5^{\circ}$ poussins en retirant chaque semaine un animal de chaque cage. En outre, les 5o poussins isolés sont habitués pendant 7 jours supplémentaires à recevoir séparément les substances azotées et l'aliment complémentaire non azoté. Pendant la période préexpérimentale, soit au total 4 semaines, les poussins reçoivent un régime complet et équilibré sauf en ce qui regarde les matières azotées qui sont constituées de tourteau de soja cru.

A la fin de la période préexpérimentale les 50 animaux sont répartis en 5 lots homogènes selon la méthode des blocs, d'après leur poids et leur gain de poids pendant la préexpérience. Les animaux reçoivent alors les régimes expérimentaux constitués par le régime protidique de base (voir tableau 2) supplémenté par la DI,-méthionine ou le DL-2-hydroxy-4-méthylthiobutyrate de calcium selon le plan expérimental qui figure au tableau $\mathrm{I}$. 
TABLEAU I

Protocole experimental

\begin{tabular}{c|l}
\hline Lots & \multicolumn{1}{|c}{ Régimes } \\
\cline { 1 - 2 } A & Régime protidique de base (R. P. B.) \\
B & R. P. B. $+0,3 \%$ de DL-méthionine \\
C & R. P. B. $+0,3 \%$ de M. H. A. \\
D & R. P. B. $+0,375 \%$ de M. H. A. \\
E & R. P. B. $+1 \%$ de DL-méthionine \\
\hline
\end{tabular}

La composition du régime protidique de base est donnée dans le tableau 2.

TABLEAU 2

Composition du régime protidique de base

\begin{tabular}{|c|c|c|}
\hline Tourteau de soja cru ....... & 88 & $\%$ \\
\hline Huile de maïs ............ & 1 & \\
\hline Complément vitaminique..... & 0,6 & \\
\hline Thréonine DI . . . . . . . . . & $0, \stackrel{9}{-}$ & \\
\hline Valine DL . . . . . . . . . . & $0,{ }^{\prime}$ & \\
\hline Lysine L . . . . . . . . . . . & 0,2 & \\
\hline Amidon q.s.p. ............ & 100 & \\
\hline
\end{tabular}

I.e complément vitaminique possède la composition qui figure dans le tableau 3 .

\section{TABLEAU 3}

Composition du complément vitaminique

\begin{tabular}{|c|c|}
\hline Composants & $\begin{array}{l}\text { Pour } 100 \mathrm{~kg} \\
\text { de régime }\end{array}$ \\
\hline 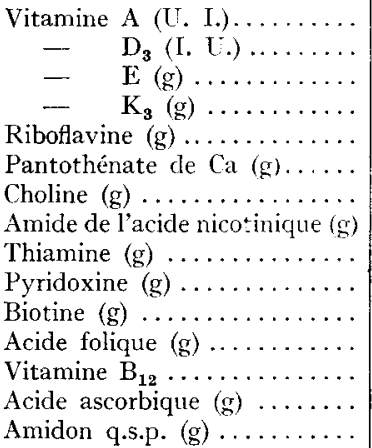 & $\begin{array}{l}1600000 \\
200000 \\
10 \\
1 \\
3 \\
3 \\
25 \\
10 \\
4 \\
2 \\
0,120 \\
0,600 \\
0,010 \\
50 \\
600\end{array}$ \\
\hline
\end{tabular}

Le régime protidique de base dose 2650 calories métabolisables par kilo et $40 \mathrm{p}$. 100 de matières azotées totales. Ces matières azotées sont déficientes en méthionine qui représente le facteur limitant du régime puisque le déficit de cet acide aminé par rapport à l'œuf est de $48 \mathrm{p}$. Ioo. C'est 
pour mieux souligner ce déficit que nous avons ajouté dans le régime protidique de base des acides aminés libres tels que thréonine, valine et lysine.

La supplémentation du régime protidique de base par la DL-méthionine ou par le DL-2-hydroxy4-méthylthiobutyrate de calcium est effectuée aux dépens de l'amidon. L'apport de M. H. A. est calculé pour fournir une quantité équivalente à la méthionine soit en produit actif soit en produit brut. L'apport de $0,3 \mathrm{p}$. 100 de cette dernière permet de ramener le déficit du facteur limitant du régime à 30 p. 100 .

Les différents régimes protidiques sont distribıés chaque jour dans une mangeoire spéciale à raison de 13 grammes par poussin. Lin outre, les animaux ont libre accès à un aliment complémentaire non azoté qui est fourni ad libitum dans une mangeoire différente. La composition de cet aliment est donnée dans le tableau 4 . Il renferme 3465 calories métabolisables par kilo.

TABLEAU 4

Composition de l'aliment complémentaire non azoté

\begin{tabular}{l}
\hline Dextrose $\ldots \ldots \ldots \ldots \ldots \ldots \ldots \ldots$ \\
Amidon $\ldots \ldots \ldots \ldots \ldots \ldots \ldots \ldots$ \\
Huile de maïs $\ldots \ldots \ldots \ldots \ldots$ \\
Cellulose $\ldots \ldots \ldots \ldots \ldots \ldots \ldots$ \\
Complément minéral $\ldots \ldots \ldots$ \\
\hline
\end{tabular}

La compnsition du complément minéral est donnée dans le tableau 5.

TABLEAU 5

Composition du complement minéral

\begin{tabular}{|c|c|}
\hline ( omposants & $\begin{array}{c}\text { Gramme } / 100 \mathrm{~kg} \\
\text { de régime }\end{array}$ \\
\hline Carbonate de calcium ....... & 1500 \\
\hline Phosphate dipotassique ..... & 730 \\
\hline Chlorure de sodium ......... & 500 \\
\hline Sulfate de magnésium, $7 \mathrm{I}_{2} \mathrm{O}$ & 500 \\
\hline Phosphate bicalcique ....... & 1700 \\
\hline Sulfate de manganèse, $\mathbf{H}_{\mathbf{2}} \mathrm{O} \ldots$ & 33 \\
\hline Citrate ferrique ............ & 33 \\
\hline Alun de potassium ......... & 1 \\
\hline Sélénite de sodium ......... & 0,055 \\
\hline Bromure de sodium, $2 \mathrm{H}_{2} \mathrm{O}$.. & 2,4 \\
\hline Chlorure de zinc ........... & 4 \\
\hline Silicate de sodium ......... & 5,5 \\
\hline Sulfate de cobalt, $7 \mathrm{H}_{2} \mathrm{O} \ldots$ & 0,2 \\
\hline Molybdate de sodium ....... & 0,9 \\
\hline Sulfate de cuivre, $5 \mathrm{H}_{2} \mathrm{O} \ldots$. & $\underline{-2}$ \\
\hline Acide borique ........... & 0,9 \\
\hline Iodure de potassium ....... & 4 \\
\hline Amidon q.s.p. . . . . . . & 5500 \\
\hline
\end{tabular}

L'eau est distribuée à volonté. L.es animaux sont éclairés I4 heures par jour. L'expérience dure 3 semaines, pendant lesquelles le contrôle hebdomadaire du poids des animaux et le relevé journalier de la consommation d'aliment sont effectués. 


\section{RÉSUITATS}

Dans le tableau 6 sont réunis les moyennes des gains de poids et des consommations des aliments pendant les trois semaines d'expérience.

TABLEAU 6

Croissance et consommation d'aliment

\begin{tabular}{|c|c|c|c|c|c|c|}
\hline \multirow[b]{2}{*}{ Lots } & \multirow[b]{2}{*}{ Régimes } & \multirow{2}{*}{$\begin{array}{l}\text { Gain } \\
\text { de } \\
\text { poids } g\end{array}$} & \multicolumn{3}{|c|}{ Consommation (g) } & \multirow{2}{*}{$\begin{array}{c}\text { Indice } \\
\text { de con- } \\
\text { sommation }\end{array}$} \\
\hline & & & $\begin{array}{l}\text { Régime } \\
\text { protidique }\end{array}$ & $\begin{array}{c}\text { Aliment } \\
\text { complémentaire } \\
\text { non azoté }\end{array}$ & $\begin{array}{c}\text { Consommation } \\
\text { totale }\end{array}$ & \\
\hline $\mathrm{A}$ & $\begin{array}{l}\text { Régime protidique } \\
\text { de base ........ }\end{array}$ & $1 / 40,8$ & 252 & 583 & 835 & 6,00 \\
\hline I3 & $\begin{array}{r}\mathrm{RPB}+0,3 \% \mathrm{mé}- \\
\text { thionine. } \ldots \ldots \ldots\end{array}$ & 235,7 & 258 & 840 & 1098 & 4,67 \\
\hline C & $\mathrm{RPB}+0,3 \% \mathrm{MHA}$ & 206,6 & 258 & 764 & $10 \div 2$ & 5,03 \\
\hline $\mathrm{D}$ & $\begin{array}{r}\mathrm{RPB}+0,375 \% \\
\mathrm{MHA} \ldots \ldots \ldots \ldots\end{array}$ & 236,7 & 259 & 824 & 1083 & 4,58 \\
\hline $\mathrm{E}$ & $\begin{array}{r}\mathrm{RPB}+1 \% \text { mé- } \\
\text { thionine....... }\end{array}$ & 267,2 & 259 & 817 & 1076 & 4,04 \\
\hline
\end{tabular}

La carence du régime protidique de base apparaitt clairement puisque tout apport de DL-méthionine est bénéfique même au taux de I p. roo Les résultats du calcul statistique effectué sur les gains de poids et les indices de consommation et qui figurent aux tableaux $7 \alpha$ et $7 \beta$ montrent que toutes les différences sont significatives sauf

TABLEAU 7

Expression statistique des résultats

3) Calculs statistiques sur les gains de poids. $\quad$ a) Calculs statistiques sur les indices de consommation

\begin{tabular}{|c|c|c|c|c|c|c|c|c|c|c|c|}
\hline $\begin{array}{c}\text { méthio- } \\
\text { nine }\end{array}$ & $\begin{array}{c}\text { méthio- } \\
\text { nine } \\
1 \%\end{array}$ & $\begin{array}{l}\text { M. H.A. } \\
0,375 \%\end{array}$ & $\begin{array}{c}\text { méthio- } \\
\text { nine } \\
0,3 \%\end{array}$ & $\begin{array}{c}\text { M.H. A. } \\
0,3 \%\end{array}$ & R.P. B. & & $\begin{array}{c}\text { méthio- } \\
\text { nine } \\
1 \%\end{array}$ & $\begin{array}{l}\text { M. H. A. } \\
0,375 \%\end{array}$ & $\begin{array}{c}\text { méthio- } \\
\text { nine } \\
0,3 \%\end{array}$ & $\begin{array}{l}\text { M.H. A. } \\
0,3 \%\end{array}$ & R. P. B. \\
\hline $\begin{array}{c}\text { méthio- } \\
\text { nine } \\
1 \%\end{array}$ & & S. & s. & HI. S. & H. S. & $\begin{array}{c}\text { méthio- } \\
\text { nine } \\
1 \%\end{array}$ & & H. S. & HI. S. & H. S. & H. S. \\
\hline $\begin{array}{c}\text { M. H. A. } \\
0,375 \%\end{array}$ & & & N. S. & S. & H. S. & $\begin{array}{c}\text { M. H. A. } \\
0,375 \%\end{array}$ & & & N. S. & H. S. & H. S. \\
\hline $\begin{array}{c}\text { méthio- } \\
\text { nine } \\
0,3 \%\end{array}$ & & & & S. & H. S. & $\begin{array}{c}\text { mèthio- } \\
\text { nine } \\
0,3 \%\end{array}$ & & & & II. S. & H. S. \\
\hline $\begin{array}{l}\text { M. II. A. } \\
0,3 \%\end{array}$ & & & & & H. S. & $\begin{array}{c}\text { M. H. A. } \\
0,3 \%\end{array}$ & & & & & H. S. \\
\hline R. P. B. & & & & & & R. P. B. & & & & & \\
\hline
\end{tabular}

H. S. = Seuil de signification, $\mathrm{P}$ inférieur à $1 \%$.

$\mathrm{S}$. = Seuil de signification, $\mathrm{P}$ inférieur à $5 \%$.

N. S. = Différence non significative. 
entre les lots qui reçoivent un supplément de $0,3 \mathrm{p}$. Ioo de DL-méthionine et de 0,375 p. Ioo de M. H. A.

En ce qui regarde l'indice de consommation les résultats sont encore plus probants.

Ainsi à égalité de produit brut, la supériorité de la DL-méthionine sur le M. H. A. est indiscutable. L'égalité de l'efficacité de ces deux produits ne se manifeste que lorsqu'on les compare sur une base équimoléculaire.

Les mêmes conciusions sont observées lorsqu'on étudie les efficacités des différents constituants de la ration. Voici dans les tableaux 8 et 9 les résultats de l'efficacité protéique (gain de poids / matières azotées consommées) et de l'efficacité calorique (gain de poids/calories métabolisables ingérées).

\section{TABLEAU $S$}

Influence de la supplémentation du rigime en méthionine et en M.H.A., sur l'efficacilé protidique du régime

\begin{tabular}{|c|c|c|c|c|}
\hline Lots & Régines & $\begin{array}{l}\text { Cain de poids } \\
(\mathrm{g})\end{array}$ & $\begin{array}{l}\text { Hatieres azoties } \\
\text { totales ingérées } \\
\qquad(\mathrm{g})\end{array}$ & $\frac{\text { Gain de poids }}{\text { Mat. azot. ingér. }}$ \\
\hline A & $\begin{array}{l}\text { Régine protidique de base on } \\
\text { K. P. B. }\end{array}$ & $1: 10,8$ & 101,:: & 1,39 \\
\hline B & R. P. B. - $-0,3 \%$ méthionine. & 235,7 & 103,3 & $\because, 98$ \\
\hline C & $-\quad+0,3 \%$ M. H. A. & 206,6 & $103,3,0$ & 2,00 \\
\hline $\mathrm{D}$ & $+0,375$ M. H. A. & $2: 36,7$ & 103,5 & 2,29 \\
\hline $\mathrm{E}$ & $+1 \%$ méthionine. & 267,2 & 103,5 & 2,58 \\
\hline
\end{tabular}

TABLEAU 9

Influence de la méthionine et du M.H.A. sur lutilisation des calories mítabolisables de la ration

\begin{tabular}{|c|c|c|c|c|c|c|c|}
\hline \multirow[b]{2}{*}{ Lots } & \multirow{2}{*}{\multicolumn{2}{|c|}{ Regimes }} & \multirow{2}{*}{$\begin{array}{l}\text { Gain } \\
\text { de poids (g) }\end{array}$} & \multicolumn{2}{|c|}{$\begin{array}{c}\text { Calories métabolisables } \\
\text { apjortées par: }\end{array}$} & \multirow{2}{*}{$\begin{array}{c}\text { Calorics } \\
\text { métabolisables } \\
\text { totales } \\
\text { consommées }\end{array}$} & \multirow{2}{*}{$\begin{array}{l}\text { Ciain de poids } \\
\text { Calories } \\
\text { métabolisables } \\
\text { consommées }\end{array}$} \\
\hline & & & & $\begin{array}{l}\text { Réginte } \\
\text { protidique }\end{array}$ & $\begin{array}{c}\text { Aliment } \\
\text { complémen- } \\
\text { taire non azoté }\end{array}$ & & \\
\hline A & \multirow{2}{*}{$\begin{array}{l}\text { R.P.13. } \\
-\end{array}$} & & $1,0,8$ & 671 & $\simeq 019$ & $=690$ & $5-1,4$ \\
\hline J3 & & $\begin{array}{c}+0,3 \% \\
\text { méthionine }\end{array}$ & $-135,7$ & bixl & 2911 & 3592 & bit, 1 \\
\hline c: & - & $+0,3 \%$ & & & & & \\
\hline 1) & - & $\begin{array}{l}\text { I.H.A. } \\
+\quad 0,375 \%\end{array}$ & $: 06,6$ & $6 \times 1)$ & 2650 & 33330 & $6: 2,2$ \\
\hline & & M.H.A. & 236,7 & $1 ; 8: 2$ & 2857 & 3539 & 66,3 \\
\hline 1 & 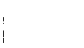 & méthionine & $\because 67,2$ & init & $\simeq 833$ & 3509 & 76,2 \\
\hline
\end{tabular}

On remarque, - sauf pour la DL-méthionine apportée au taux de I p. Ioo l'évolution parallèle entre le développement pondéral et la libre consommation de calories non azotées. Il en résulte que la consommation totale d'énergie s'élève au fur et à mesure de l'accroissement de l'efficacité du régime. 
On remarque également la relation étroite qui existe entre l'augmentation des efficacités protéiques et caloriques lorsque la croissance des animaux est améliorée.

\section{DISCUSSION}

Les études qui ont été entreprises pour démontrer l'intérêt du M. H. A. ont été conduites dans un but essentiellement pratique. Pour cela, les auteurs ont utilisé des régimes commerciaux à base de maïs et de soja dont le taux protéique était conforme aux normes (20 à $2 \mathrm{I}$ p. Ioo de matières azotées totales). D'une manière plus fine, BIRD en I952 utilise un régime semi-synthétique à base de tourteau d'arachide dosant également environ $20 \mathrm{p}$. Ioo de protéines. A quantité équivalente de produit actif, la méthionine, le M. H. A. sous forme d'amide ou sous forme de sel de calcium ont a peu près la même activité aux différents taux où on les compare. Ces résultats sont confirmés par GordoN et SIZER, I955.

On peut reprocher à ces travaux, comme l'ont fait SULIIVAN et BIRD, I957, de manquer de sensibilité pour mettre en évidence les éventuelles différences entre II. H. A. et méthionine en raison du taux protéique trop élevé des régimes. Lorsque ces derniers renferment I 2 ou I 4 p. Ioo de matières azotées déficientes en acides aminés soutrés (protides purifiées de soja), on observe une infériorité constante du M. H. A. vis-à-vis de la DL-méthionine comparés molécule à molécule. Plus récemment, nous avons confirmé ce résultat en utilisant des régimes à base de soja cru dosant I6 p. Ioo de matières azotées (CALET et BARATOU, I960). Il convient de citer une seule exception à cette manière de voir qui a été signalée par MACHELIN et Gordon, I959. Avec des rations dosant I4 p. Ioo de protéines, ces auteurs observent une action égale du M. H. A. et de la méthionine lorsque la mesure commune de ces corps est leur poids brut; on ne s'explique pas les raisons de cette exception.

En utilisant une technique inhabituellement pratiquée, nous confirmons une fois de plus 1'opinion généralement admise. Le plan de rationnement que nous avons appliqué donne au poussin la possibilité d'ingérer librement la quantité de substances énergétiques qui lui convient et lui permet d'utiliser au mieux les matières azotées du régime. De plus, lorsque l'on considère la totalité des ingérés du poussin (somme des consommations du régime protidique et de l'aliment complémentaire non azoté), on s'aperçoit que les an.maux des différents lots se sont constitués des régimes qui dosent environ io $\mathrm{p}$. roo de matières azotées totales. Nous nous sommes donc placés dans les conditions les plus favorables pour évaluer les efficacités des produits étudiés.

Nos résultats indiquent que le nombre de calories librement consommées diffère selon la nature du mélange azoté et nous constatons en outre que le M. H. A. et la méthionine, apportés en quantités équivalentes, conduisent à la même croissance, à la même efficacité protidique et à la même consommation de calories. En revanche, comparés sur la base du produit brut, le M. H. A. se montre toujours inférieur quel que soit le critère choisi.

En résumé, nous retrouverons une fois de plus que le $\mathrm{M}$. H. A. ne peut prétendre à posséder une efficacité pour la croissance, égale à celle de la DL-méthionine, lorsqu'on les compare sur la base du poids de substance brute. L'équivalence ne peut exis- 
ter entre ces produits que sur la base du produit actif. Ces résultats sont vrais pour la croissance, pour l'indice de consommation, et pour les efficacités protidique et calorique du régime.

\author{
Reçu en octobre 1961.
}

\title{
SUMMARY
}

COMPARISON OF EFFICIENCY FOR GROWTH OF METHIONINE

AND CALCIUM IYDROXYMETHYLTHOBLTYRATE (M. H. A.).

The ability of a protein in promoting growth is closely dependent on the energy content of the diet. It has been previously clemonstrated (CALET, JOCANDET, BARATOU, 1960) that the optimal amount of calories per gram of protein ingested is related to the nature of the dietary protein. The higher the biological value of the protein, the greater the amount of calories ingested.

In order to demonstrate clearly the differences in efficiency between two proteins, it appeared necessary to formulate diets differing in their energy content. After IllLL and DANSKY, 1954, we know that the chick is able to regulate its calorie intake.

Chicks were supplied simultaneously in two separate feeders with a protein dict, and a comple mentary nitrogen-free diet. The protein diet contained to p. 100 raw soybean protein supplemented with lysine, threonine and valine and included the adequate vitamins. It was given in restricted amount, $830 \mathrm{mg}$ per bird and per day. The complementary nitrogen-free diet contained carbohydrates, fat and minerals. It was distributed ad libitum in order to let the chick satisfy its energy requirenent. The experiment was carried out on 4 week old Rhode-Wyandotte chicks in individual cages and lasted $2 \mathrm{I}$ davs.

Ender these conditions, the ability of DL-methionine to improve raw soybean meal was compared to that of calcium-DL-hydroxymethylthiobutyrate.

From our results, it is concluded that :

I) The protein diet is greatly deficient in sulfur amino acids, as evidenced by the beneficital effect produced by i p. 100 additional methionine.

2) The spontaneous energy intake is related to the protein efficiency.

3) When DL-methionine $(0,3 \mathrm{p} .100)$ and I. II. A. (0,375 p. 100) are compared on a molar basis, identical growth, spontaneous energy consumption, and protein and energy efficiency were observed.

4) When, on the other hand, the comparison is made on a crude product basis, $(0,3 \mathrm{p}$. 100) M. 11. A. is always inferior to methionine, whatever the evaluation criterium used.

M. Il. A. contains $80 \mathrm{p}$. 100 active product, while DI-methionine counts $98 \mathrm{p}$. 100 . In no case can M. H. A. pretend to have the same efficiency as methionine when compared on the crude product basis.

\section{RÉFÉRENCES BIBLIOGRAPHIQUES}

Bartox L., Stephexistox E. L., Ig60. Dietary interrelationships between levels of protein, fat, and supplementary methionine hydroxy analogue for both egg type and meat type hens. Poul.. Sci., 39, I 233.

BIRD F. H., I952. A comparison of methionine and two of its analogues in the nutrition of the chick. Poult. Sci., 31, $1095^{-1090 .}$

Calfe C., Baratou J., ig60. Comparaison de l'eficacité pour la croissance du poussin de la méthionine et des hydroxyanalogues de la méthionine. Les industries de l'alimentation animale, $\mathrm{n}^{\circ} \mathrm{I} 07,4 \mathrm{I}-49$.

Calet C., Jouandet C., Baratol J., ig6r. Variation de la consommation spontanée d'énergie du poussin en fonction de la nature des matières azotées du régime. Ann. Biol. anim. Bioch. Biophys., 1, 5-9.

COHEN H. P., CinolTz H. C., BerG C. P., i958. Response of rats to dicts high in methionine and related compounds. J. Nutr., 64, 555-569.

Douglas C. R., Hochreich H. J., Harms R. H., 1957. Cilycine and méthionine supplementation of practical broiler feeds. Poult. Sci, 36 , I 14 .

GORDON R. S., MADDY K. H., KNIGHT S., I954. Value of methionine hydroxyanalogue supplementation of broiler rations. Poutl. Sci, 33, 424-425.

Gordon R. S., SIzer I. W., 1955. The biological equivalence of methionine hydroxy-analogue. Poult. Sci., 34, I 198 . 
Gordon R. S., Machlis I. J., MADDY K. II., I957. Replarement of protein of corn-soybean diets with amino-acids. Poult. Sci., 36, I 122.

Hill I. W., DANSky L. M., I954. Studies on the energy requirements of chickens. I. - The effect of dietary energy level on growth and feed consumption. Poult. Sci., 33, I z-I I9.

Macilin L. J., Gordon R. S., I958. Rapid growth of chickens fed an improved semi-synthetic diet. Poull. Sci., 37, I460-1461.

Machins L. J., GoRdon R. S., 1959. Equivalence of methionine hydroxy analogue and methionine for chickens fed low-protein diets. Ponlt. Sci., 38, 650-652.

MAW G.A., du Vigneaud V., I948. Dimethyl \&-propiothetin a new methyl donor. J. Biol. Chem. 174, 381 382.

REID B. L., Gabay S., Couch J. R., I954. Effect of methionine, methionine hydroxy-analogue and vitamine $\mathrm{B}_{12}$ on the glutathion level in the chick. Poult. Sci., 33, rogo.

Sanson W. R., Matterson L. D., 5957 . The use of dimethyl $\beta$-propiothetin as a substitute for supplenental methionine in a purified diet deficient in sulphur amino-acids. Potlt. Sci., 36, 41 I-4I3.

Sullivan T. W., BIRD H. R., I957. Effect of quantity and source of dietary nitrogen on the utilization of the hydroxy-analogues of methionine and glycine by chicks. J. Nutr., 62, I $43^{-1} 50$. 